\title{
Nanocarreadores aplicados ao tratamento do câncer de mama
}

\author{
Nanocarriers applied to the treatment of breast cancer \\ Nanoportadores aplicados al tratamiento del cáncer de mama
}

\section{Resumo}

Objetivo: Avaliar as implicações da utilização dos nanocarreadores no tratamento do câncer de mama. Métodos: Revisão da literatura com base nas diretrizes contidas no Preferred Reporting Items for Systematic reviews and Metaanalyses (PRISMA), utilizando as bases de dados: PubMed e Biblioteca Virtual em Saúde com os termos de busca: "nanopartículas" E "tratamento" E "câncer de mama". Foram selecionados 19 artigos, escritos em inglês, que preencheram os critérios de inclusão. Resultados: Diante dos achados nos estudos selecionados, percebeu-se que os nanocarreadores apresentam baixa toxicidade a células saudáveis e as nanopartículas podem ser direcionadas para o local específico do tumor. Conclusão: este estudo permitiu compreender a eficácia das drogas utilizadas nos diferentes tipos de nanocarreadores para a redução das células tumorais com minimização da toxicidade e liberação do medicamento na região específica do tumor.

Palavras-chave: Nanopartículas; Tratamento; Neoplasias de mama.

\begin{abstract}
Objective: Evaluate the implications of using nanocarriers in the treatment of breast cancer. Methods: Literature review based in guidelines contained in Preferred Reporting Items for Systematic reviews and Meta-analyses (PRISMA), using these databases: PubMed and Biblioteca Virtual em Saúde, using the key words: "nanoparticles" AND "treatment" AND "breast cancer". Nineteen articles, written in English, that met the inclusion criteria, were selected. Results: Given the findings on the selected studies, it was perceived a low toxicity on healthy cells and the capacity to direct nanoparticles to the tumor site. Conclusion: The present study allowed the comprehension of the efficacy of de drugs
\end{abstract}


carried on the different nanoparticles, intending to reduce the tumoral cells and minimize the toxicity, with specific liberation of drugs on tumor site.

Keywords: Nanoparticles; Treatment; Breast neoplasms.

\section{Resumen}

Objetivo: Evaluar las implicaciones del uso de nanoportadores en el tratamiento del cáncer de mama. Metodología: Revisión de la literatura basada en las pautas contenidas en los elementos de informe preferidos para revisiones sistemáticas y metaanálisis (PRISMA), utilizando las bases de datos: PubMed y Virtual Health Library con los términos de búsqueda: "nanopartículas" $Y$ "tratamiento" $Y$ "cáncer de mama". Se seleccionaron diecinueve artículos, escritos en inglés, que cumplieron con los criterios de inclusión. Resultados: Con base en los hallazgos de los estudios seleccionados, se observó que los nanoportadores tienen baja toxicidad para las células sanas y que las nanopartículas pueden dirigirse a la ubicación específica del tumor. Conclusíon: este estudio permitió comprender la efectividad de los fármacos utilizados en diferentes tipos de nanoportadores para la reducción de células tumorales con minimización de la toxicidad y liberación del fármaco en la región específica del tumor.

Palabras clave: Nanopartículas; Tratamiento; Neoplasias de la mama.

\section{Introdução}

O câncer de mama $(\mathrm{CM})$ é uma doença causada pela multiplicação desordenada de células da mama. Esse processo gera células anormais que se multiplicam, formando um tumor. Há vários tipos de $\mathrm{CM}$, que podem evoluir e se desenvolver de diferentes formas (INCA, 2021). O CM surge nas células de revestimento (epitélio) dos ductos (85\%) ou lóbulos (15\%) no tecido glandular da mama. Com o tempo, os cânceres in situ (estágio 0) podem progredir e invadir o tecido mamário circundante (câncer de mama invasivo) e se espalhar para os linfonodos próximos (metástase regional) ou para outros órgãos do corpo (metástase distante) (OMS, 2020).

De acordo com a Organização Mundial de Saúde, em 2020, havia 2,3 milhões de mulheres com diagnóstico de câncer de mama e 685 mil mortes em todo o mundo. No final de 2020, havia 7,8 milhões de mulheres vivas com diagnóstico de câncer de mama nos últimos 5 anos, tornando-se o câncer mais prevalente do mundo (OMS, 2020). O tratamento do câncer de mama pode ser altamente eficaz, especialmente quando a doença é identificada precocemente. No entanto, embora esforços sejam obtidos nos tratamentos convencionais incluindo cirurgia, radioterapia, quimioterapia, hormônio terapia e imunoterapia, a metástase do câncer de mama revela um cenário conturbado. (Siegel, 2012). De forma geral, as estatísticas indicam que a disseminação mundial do CM é significativa. Isso forneceu o ímpeto para a investigação da biologia do CM para identificar aberrações associadas à malignidade, marcadores de diagnóstico clínico e potenciais alvos para terapia. (Padayachee, 2020).

Desafios são encontrados no tratamento da metástase do câncer de mama, como a agressiva proliferação do tumor, a metástase para múltiplos órgãos, resistência à terapêutica aplicada, heterogeneidade dentro das metástases e a barreira hematoencefálica, a qual proporciona invasão para o tecido cerebral (Jones, 2008; Lu, 2009). Estratégias de tratamento multimodal, incluindo uma combinação de cirurgia, radioterapia e quimioterapia não conseguiram melhorar os resultados do tratamento em casos de câncer de mama metastático. A quimioterapia convencional tem a limitação devido à distribuição não seletiva de drogas anticâncer entre as células cancerígenas e as células saudáveis. (Maji, 2014; Pourjavadi, 2018). Com base nisso, o principal desafio do tratamento do câncer é a distribuição de substâncias tóxicas em locais específicos com efeito colateral mínimo em tecidos e órgãos saudáveis. Nos últimos anos, várias pesquisas em terapias baseadas em nanopartículas têm sido realizadas como uma abordagem alternativa, em que os nanocarreadores, definidos como fármacos anticâncer citotóxicos carregados em nanopartículas que podem ser direcionadas para o local específico do tumor usando um campo magnético externo com efeitos colaterais mínimos (Panda, 2020).

A engenharia do nanocarreador (NP) é empregada para aumentar a estabilidade, especificidade e eficiência de sistemas de entrega de ácido nucleico não viral. (Padayachee, 2020). Uma vantagem da utilização de NPs é que eles fazem uso de materiais de ocorrência natural ou sintéticos que podem eliminar as respostas imunes, como fármacos e genes, apresentando baixa toxicidade a células saudáveis e alta especificidade ao tumor, direcionando a liberação dos agentes terapêuticos. Devido à 
superfície de NP imuno quimicamente inerte, o tempo de retenção da formulação in vivo é aumentado em consequência da diminuição na enzima de degradação e sequestro por fagócitos, provendo uma vantagem à utilização destes sistemas (Padayachee, 2020). Nesse sentido, esta revisão objetivou avaliar as implicações, eficácia e segurança de nanocarreadores mais utilizados no tratamento do câncer de mama.

\section{Metodologia}

A pesquisa tem natureza qualitativa (Estrela, 2018). A estratégia de busca foi baseada nas Preferred Reporting Items for Systematic Reviews and Meta-Analyses (PRISMA) guidelines. Os artigos foram identificados e selecionados para leitura e seleção dos estudos para inclusão. A busca foi realizada nas bases de dados PubMed e Biblioteca Virtual em Saúde. Foram utilizados os descritores e Medical Subject Headings (MeSH) "nanocarriers", "treatment", "breast cancer" com o operador Booleano "AND". Os artigos incluídos tratam abordagens que se relacionam com o tema, foram publicados entre os anos 2016 e 2021, disponibilizados em inglês. A pesquisa foi iniciada em Fevereiro de 2021. A Figura 1 mostra a estratégia de busca.

Figura 1 - Fluxograma do PRISMA.
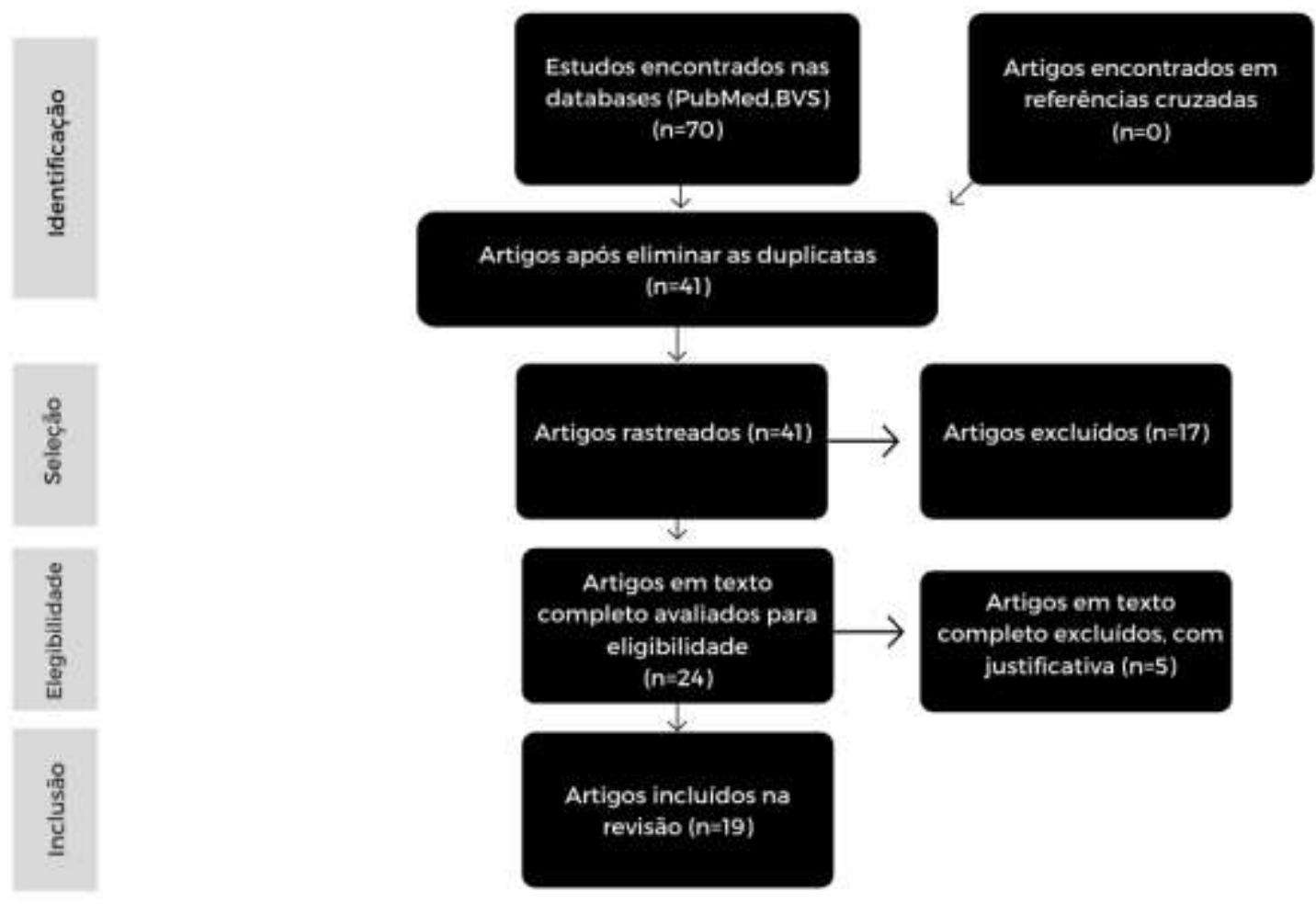

Fonte: Autores (2021).

Utilizou-se o aplicativo Rayyan Qatar Computing Research Institute (QCRI) como uma ferramenta auxiliar para exclusão, seleção e organização dos artigos. Os critérios de inclusão utilizados foram: 1) Pesquisas originais que relatam a eficácia do uso de nanocarreadores no tratamento do câncer de mama. 2) Artigos escritos na língua inglesa. 3) Período de publicação de 2016 a 2021. Os critérios de exclusão foram: 1) Artigos duplicados. 2) Artigos não relacionados diretamente ao tema.

Os dados coletados pelos revisores foram o título, autor, ano e localização. Em adição, informações sobre o número amostral, o nanocarreador utilizado, resultados e os pontos principais foram coletados. Dois revisores independentes avaliaram 
e classificaram os artigos de acordo com suas qualidades. Os dados dos artigos foram extraídos e inseridos dentro de uma tabela, utilizando o Planilhas Google, para análise pelos revisores.

\section{Resultados e Discussão}

A pesquisa inicial resultou em 70 artigos potencialmente relevantes. Foram excluídas as duplicatas, sendo qualificados 21 artigos para análise. Com base nos resumos disponíveis, foram selecionados 19 artigos para leitura integral, todos correlacionados aos critérios de inclusão previamente propostos (Quadro 1). Dentre os estudos levantados, os trabalhos eram provenientes de periódicos internacionais, escritos em inglês. O risco de viés dos artigos foi avaliado pelo RevMan (Figura 2).

Figura 2 - Tabela do Risco de Viés.

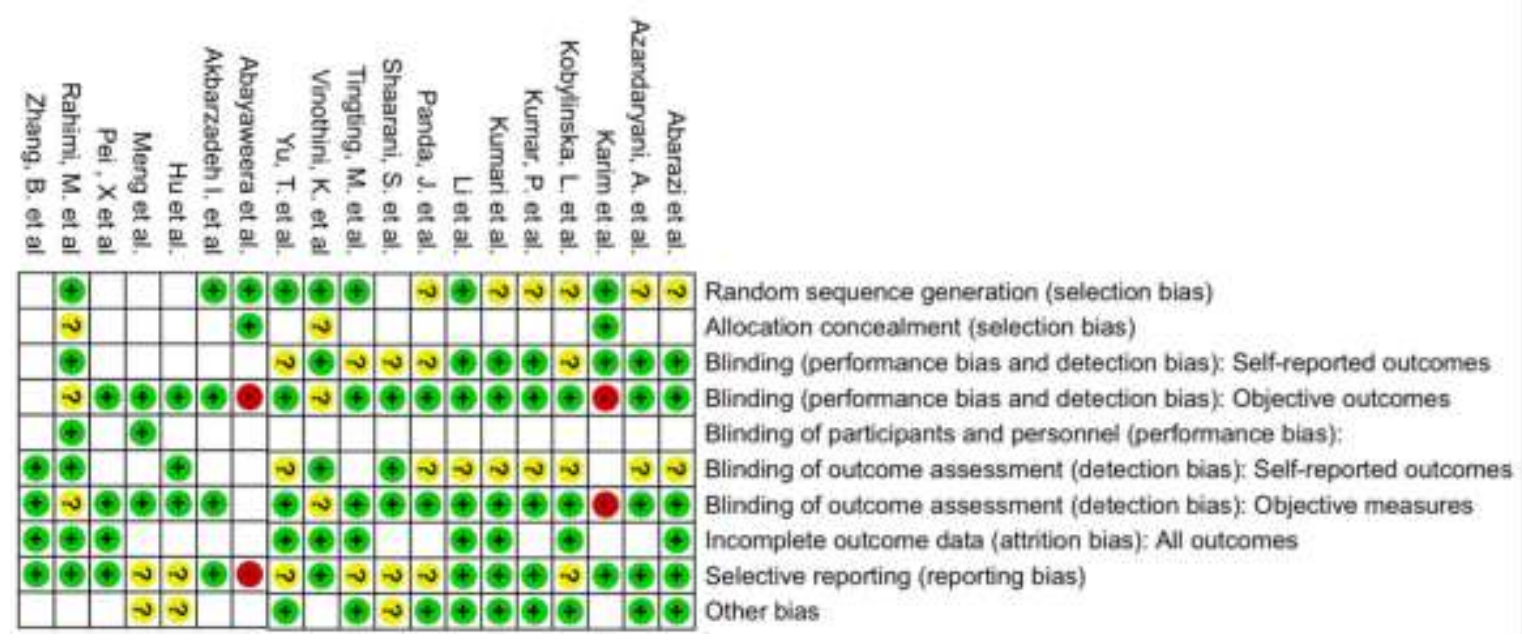

Fonte: RevMan Cochrane (2021).

\subsection{Nanopartículas Poliméricas}

As nanopartículas poliméricas (NPPs) têm sido amplamente utilizadas na área farmacêutica para encapsulação de princípios ativos. Essas nanopartículas apresentam as vantagens de proteção, liberação controlada, melhor biodisponibilidade e menor toxicidade, proporcionando maior conforto aos pacientes e adesão ao tratamento. As NPPs podem ser produzidas a partir de diferentes componentes sintéticos ou naturais, como quitosana, PNC, Niossoma-cálcio alginato, timoquinona, ferrita de cobalto.

Azandaryani (2017) estudou a afinidade da quitosana para o fármaco letrozole (LTZ). A incorporação de lipídios ao complexo levou a um aumento da afinidade do fármaco com o nanocarreador. O perfil de liberação in vitro de LTZ nos testes de precipitação demonstrou a liberação controlada de LTZ pelo transportador híbrido FA-PLNs (ácido fólico-nanopartículas de lipídios sólidos revestidos) com meia-vida adequada aumento no corpo. Para FA-PLNs, a liberação cumulativa alcançou quase até $90 \%$ em 72 h. De acordo com os resultados de citotoxicidade da linhagem celular, a eficiência dos FA-PLNs para a captação intracelular de LTZ foi comprovada como resultado da endocitose mediada pelo receptor de FA para o tratamento do câncer de mama.

Kumari (2017) evidenciou que o tratamento com CUR-mPEG-PLA-Ch foi mais eficaz em matar células cancerígenas, embora em menor proporção no período de tempo menor que $24 \mathrm{~h}$ em células B16F10. Isto pode ser devido à liberação incompleta do fármaco carregado da formulação, resultando em uma menor eficácia no tratamento. Além disso, estudos anteriores por outros grupos indicaram que o valor de IC50 variou em diferentes linhas de células com o mesmo tratamento 
Kobylinska (2018) demonstrou a eficiência da liberação de NPs de doxorrubicina, de preparação antitumoral contendo rutênio KP-1019, e de derivados experimentais anticâncer de 4-tiazolidinona, todos voltados para inibir o crescimento e a sobrevivência de células tumorais in vitro e in vivo em ratos e camundongos de laboratório. Akbarzadeh (2021), no Irã, realizou estudos de citotoxicidade in vitro que mostraram alta compatibilidade de AL-NioC (Niossoma-cálcio alginato) com células normais MCF10A, enquanto toxicidade significativa foi observada em células de câncer de mama MDA-MB-231 e SKBR3. Estudos de citometria de fluxo confirmaram um aumento significativo na taxa de apoptose na presença de AL-NioC em ambas as células MDA-MB-231 e SKBR3 em comparação com outras amostras.

Shaarani (2017) mostrou em um estudo in vitro, na Malásia, que os diferentes pesos moleculares e número de segmentos hidrofílico-hidrofóbicos do Pluronic mostraram resultados significativos nas propriedades físico químicas, liberação in vitro, e efeito antiproliferativo do fármaco TQ em relação às células cancerosas MCF-7. Pode-se concluir que um peso molecular mais alto com segmentos hidrofílico-hidrofóbicos mais longos de micelas de polímero Pluronic (F127) pode ser usado como um candidato adequado para a entrega sustentada de drogas.

\subsection{Nanopartículas Metálicas}

O nanocarreador de ferrita de cobalto (CF-NP) biocompatível foi desenvolvido com sucesso para a entrega sustentada e específica de células de docetaxel (DTX) para células de câncer de mama que mostraram extensa distribuição intracelular em ambos os tipos celulares cancerígenos (MCF-7 e MDA-MB-231) (Panda, 2020). Sua natureza antiproliferativa contra as células de câncer de mama humano evidencia sua potência para aplicação clínica. Ademais, a toxicidade insignificante para as células sanguíneas reflete sua natureza segura para o homem. Todos esses resultados sugerem a aplicação potencial de DCF-NP para a entrega de DTX para tratamento de câncer de mama.

Abarazi (2018), analisou as imagens de microscopia de fluorescência do controle Células MCF-7 tratadas. Esclareceram que a DOX carregada positivamente com CS / Bio-MOFs interagem com a célula carregada negativamente e utilizam membranas para se difundir nas células cancerosas e liberar DOX, sob a condição ácida de tumores. Lá, a DOX lançada às moléculas podem se localizar nos núcleos das células e induzir a apoptose eficientemente. Desta forma, os CS / Bio-MOFs carregados com DOX exibem uma maior extensão da apoptose celular. Em geral, nos estudos in vitro os resultados mostraram que o Bio-MOF-13-Co modificado de superfície desempenham maior especificidade e citotoxicidade em relação ao material original, além de atuar como responsivo ao $\mathrm{pH}$ portador que pode resultar em uma maior eficácia no tratamento de câncer com menos efeitos colaterais.

Nos Estados Unidos, analisaram nanocarreadores de ferro ( $\mathrm{Fe} / \mathrm{Fe} 3 \mathrm{O} 4)$ e enxofre em células de câncer de mama cultivadas. Nos desfechos, observaram o potencial de levar a uma nova geração de nanoplataformas para o tratamento do câncer com seletividade consideravelmente aumentada para células tumorais (Abayaweera, 2017).

\subsection{Nanopartículas Biológicas}

Nanocarreadores são capazes de reter a droga no pH fisiológico $(7,4)$ enquanto a libera em pH mais baixo $(5-6,5)$, cujo os locais do tumor são fortemente preferidos. O nanocarreador de doxorrubicina (DOX) carregada por Triptofano (PTN) mostrou liberação rápida de $\mathrm{DOX} \mathrm{em} \mathrm{pH} \mathrm{5,} \mathrm{mas} \mathrm{em} \mathrm{pH} \mathrm{7,4,} \mathrm{foi} \mathrm{observada} \mathrm{liberação} \mathrm{bifásica} \mathrm{(liberação} \mathrm{repentina} \mathrm{seguida} \mathrm{de} \mathrm{liberação}$ sustentada). Essa liberação prolongada da droga pode ser atribuída à presença de triptofano que causam maior tensão no obstáculo (Kumar, 2018).

Os tumores podem se tornar resistentes a uma ampla gama de drogas anticâncer, levando a múltiplas drogas resistência (MDR). Uma vez que a MDR aparece, uma dose ainda mais alta do medicamento é ineficaz e levará a mais toxicidade severa. O mecanismo mais comum de resistência envolve o efluxo de drogas das células por transportadoras de ligação de adenosina 5'trifosfato (ATP). O transportador mais amplamente envolvido, a glicoproteína P (gp-P), é expressa em muitos cânceres humanos, 
incluindo câncer de mama. A P-gp pode detectar e ligar uma grande variedade de drogas à medida que entram na membrana plasmática e, em seguida, excreta-las para fora da célula, contribuindo para a redução do acúmulo intracelular de drogas e comprometimento da eficácia da droga. O nanocarreador semelhante a glicolipídeo à base de quitosana (CSOSA) carregado com doxorubicina (DOX) mostrou grande potência para contornar a quimiorresistência à base de efluxo de gp-P. Isso porque, não se liga a domínios transmembranas (TMDs) e contorna o efeito de efluxo por Pgp. Embora a P-gp tivesse excelente flexibilidade estrutural, que ajudava a acomodar uma certa quantidade de substratos de várias formas e tamanhos, os substratos eram limitados àqueles com massa variando de aproximadamente 300 a $4000 \mathrm{Da}$. A molécula do fármaco DOX era um substrato, porém estava encapsulada na CSOSA que era formada por várias cadeias de quitosana, sendo o tamanho e peso molecular muito grandes para serem reconhecidos pela TMDs (Tingting, 2018).

Um siRNA de regulação negativa de P-gp (siMDR1) e doxorrubicina (DOX) foi utilizado para eliminar células de câncer de mama multirresistentes (MCF-7 / ADR). Um nanocarreador de glicolipídeo responsivo a redox (CSO-ss-SA) foi usado para condensar siRNA (CSO-ss-SA / siRNA) e encapsular DOX (CSO-ss-SA / DOX) separadamente. Eles responderam ao ambiente de alta redução das células tumorais e rapidamente liberaram a carga útil. CSO-ss-SA / siMDR1 silenciou o gene MDR1 e resultou em uma diminuição transitória da P-gp. Sequencialmente, a formulação DOX (CSO-ss-SA / DOX ou DOX . $\mathrm{HCl}$ ) foi entregue quando a P-gp foi reduzida ao nível mais baixo. Após o pré-tratamento por CSO-ss-SA / siMDR1, a citotoxicidade de CSO-ss-SA / DOX e DOX · HCl contra células MCF-7 / ADR foi de 6,4 ou 3,4 vezes, respectivamente, daquela tratada por $\mathrm{DOX} \cdot \mathrm{HCl}$ sozinho, que exibiu aumento sensibilidade à citotoxina de células resistentes a drogas e resultados terapêuticos maximizados (Meng, 2017).

A superexpressão da proteína Bcl-2 está relacionada à resistência aos medicamentos e ao mau prognóstico em pacientes com câncer. Um sistema de entrega sequencial de droga dependente do tempo (DDS) com base em um nanocarreador à base de quitosana ligado por dissulfeto (CS-ss-SA) foi desenvolvido para a co-entrega de paclitaxel (PTX) e siRNA específico de Bcl-2 (siBcl-2). Este nanocarreador CS-ss-SA é capaz de transportar drogas e siRNA por aprisionamento de PTX e adsorção de siRNA por atração eletrostática, ele transporta siRNA em células tumorais por meio de sua estrutura espacial semelhante a glicolipídeo. Quando o siRNA e a co-entrega de PTX e siBcl-2 por CS-ss-AS às células tumorais, um efeito sinérgico foi observado tanto na parada do ciclo celular quanto na viabilidade celular (Hu, 2016).

\subsection{Nanopartículas Lipossomais}

Li e colaboradores, em 2019, realizaram um estudo de um sistema de co-entrega lipossomal encapsulando DOX e PT (ácido paquímico e ácido desidrotumulósico), sendo este os dois principais componentes triterpenóides purificados e identificados no fungo poria cocos. Esse sistema foi utilizado como recurso terapêutico para reverter a resistência a múltiplas drogas (MDR), frequentemente induzida pelos tratamentos de quimioterapia convencional que utilizam drogas tóxicas, limitando os resultados da quimioterapia. Segundo os autores, uma terapia com combinação poderia sensibilizar o tumor às medicações, modificar diferentes células cancerígenas e aceitar a entrega de doses menores de cada droga, diminuindo efeitos adversos e contribuindo em um melhor resultado clínico. O estudo propôs construir um sistema "tudo em um", que entregaria DOX e extrato de triterpenóides de PT em um transportador lipossomal (DOX-PTL), com o PT como um reversor de MDR com melhor segurança que medicamentos quimioterápicos convencionais e a DOX para atingir células tumorais especificamente nos tumores resistente a drogas, além disso, o nanossistema de lipossoma melhorando o efeito de permeabilidade e retenção (EPR) no ambiente tumoral, aumentando o direcionamento de DOX e PT aos tumores, o que reduz os efeitos tóxicos em células saudáveis.

Em in vitro, os resultados de citotoxicidade mostraram que a co-entrega de lipossomas resultaria no benefício antitumoral. Fazendo o comparativo com recentes relatórios de combinações de quimioterápicos, não foram observadas toxicidades óbvias, demonstrando segurança no design "tudo em um" com PT e DOX. Esse sistema contribuiu de forma significativa no 
efeito antitumoral de DOX em camundongos com câncer em relação a diferentes grupos de tratamento exclusivo. As respostas adquiridas fundamentam atitudes estratégicas no recurso terapêutico de co-entrega para o tratamento de câncer (Li, 2019).

\subsection{Nanopartículas Micelares}

Yu e colaboradores (2020) desenvolveram um nanocarreador a base de ácido hialurônico (AH) incorporando DOX e cisplatina (CDDP) como um composto de aplicação de medicamentos anti-câncer, sendo direcionada a células CD44+. O estudo realizado demonstrou que as micelas de $\mathrm{AH}$ contendo uma droga dupla (AH-DOX-CDDP) foram liberadas de forma consideravelmente maior em condições de $\mathrm{pH}$ ácido, além de demonstrarem uma inibição do crescimento celular maior do que medicações livres contra células cancerígenas de mama 4T1, que expressam altos níveis de receptores CD44. O composto AHDOX-CDDP teve taxas de liberação de DOX que aumentaram de acordo com a diminuição do pH, esse resultado foi atribuído à cisão de grupos carboxila entre AH e DOX sob condições ácidas, tal sensibilidade ocasiona uma liberação mais rápida do medicamento no ambiente tumoral, aumentando sua ação anti-tumoral. Além disso, a droga AH-DOX-CDDP apresentou entrega direcionada às células que expressam altos níveis de CD44, ação devida à interação do medicamento com receptores CD44 na superfície das células 4T1. Tais descobertas demonstram que micelas de AH carregadas com DOX exibem um direcionamento ideal mediado por CD44 e liberação dependente do pH no microambiente do tumor, o que pode resultar na diminuição dos efeitos colaterais e aumento da eficiência do fármaco.

As micelas de AH-DOX-CDDP, analisadas em um modelo de camundongo com câncer de mama, demonstraram menor toxicidade e melhores efeitos inibitórios do que drogas livres, de acordo com os testes utilizando imunofluorescência e análise histológica. Esse resultado demonstra que o composto AH-DOX-CDDP representa um sistema de medicamento animador, com excelente biocompatibilidade e biodegradação, relatando a promissora aplicação dessa medicação em quimioterapia para câncer de mama (Yu, 2020).

\subsection{Nanopartículas Orgânicas}

O nanocarreador de óxido de grafeno-acrilato de metila conjugado com ácido fólico (GO-g-MA/FA) foi testado por Vinothini e colaboradores (2019). Foi analisado o perfil de liberação da droga, tendo sido mostrada uma boa eficácia de liberação (71\%) em ambientes com pH de 2,8, similares aos microambientes ácidos encontrados nos tumores de mama, enquanto em ambientes fisiológicos e levemente ácidos, sua liberação foi baixa (pH7,4 - 48\%; pH5,5 - 65\%). A citotoxicidade foi testada in vitro, em células de câncer de mama MDA-MB-231. Nos grupos controle e utilizando CO-g-MA/FA não carregado, não foram apresentadas diferenças significativas visíveis. Quando analisados os grupos com diferentes níveis de PTX, evidenciou-se apoptose celular. A citotoxicidade demonstrou-se concentração-dependente, sendo maior nos grupos testados com 100 $\mu \mathrm{g} / \mathrm{mL}-1$ de PTX (39\%). O IC50 determinado foi de $75 \mu \mathrm{g} / \mathrm{mL}-1$.

Os potenciais anticâncer do OG-g-MA/FA carregado com PTX foi testado em ratos com câncer de mama induzido por DMBA. Nos ratos que não receberam tratamento, a perda de peso foi substancial, quando comparados aos ratos do grupo controle. Nos ratos em que foi administrado o PTX carregado pelo nanocarreador ( $5 \mu \mathrm{g} / \mathrm{Kg}$ peso corporal/semana) houve evidente recuperação de peso e diminuição do tumor, comparados com os ratos com o tumor induzido por DMBA e os tratados apenas com o nanocarreador. Os níveis de enzimas da cadeia respiratória e do ciclo do ácido cítrico dos ratos tratados com o OG-gMA/FA com PTX apresentaram recuperação, similares aos normais, enquanto o grupo com o câncer DMBA-induzido apresentou significativa redução dos níveis enzimáticos. Marcadores enzimáticos apresentaram-se baixos, similarmente aos níveis normais. Com os testes histológicos, foi apresentada grande biocompatibilidade, reduzidos efeitos adversos do medicamento e um potencial uso anticâncer. (Vinothini, 2019). 
Pei e colaboradores (2020) desenvolveram um nanocarreador de óxido de grafeno PEGuilado (pGO) duplamente carregado com cisplatina (Pt) e doxorrubicina (DOX). Com a proporção de massa otimizada de 0,367:0,367:1 de DOX:Pt:pGO, foi identificada um desprendimento das drogas $\mathrm{pH}$-dependente, com eficácia na entrega tumoral. Além disso, foram mostradas diminuições nos efeitos colaterais, com diminuição da toxicidade sistêmica da Pt e DOX combinadas com o nanocarreador, em comparação às drogas livres. Os efeitos anticâncer foram observados com excelência nos testes com o uso do tratamento de dupla droga carregadas pelo nanocarreador.

O Nanocarreador composto de polifosfato éster (NP) com agente fototérmico hidrofóbico IR-780 e curcumina radiossensibilizante (Cur) foi desenvolvido por Zhang e colaboradores (2019). Seu efeito fototérmico foi testado, sendo medido por uma câmera infravermelha, com a irradiação de um laser de $808 \mathrm{~nm}$ de comprimento de onda. Nos grupos onde havia uma solução de tampão fosfato-salino apenas, houve um aumento de $1,2^{\circ} \mathrm{C}$, enquanto nas soluções contendo o NP/IR e NP/IR+Cur os aumentos se mostraram dose-dependentes e suficientes para o tratamento fototérmico do câncer in vivo. A captação de curcumina foi estudada em células MDA-MB-231, com curcumina livre, NP/IR+Cur e NP/Cur em 2, 6 e 12 horas. Com o uso de cromatografia líquida de alta eficiência foi observado um aumento gradual na concentração de curcumina nas células incubadas com NP/IR+Cur e NP/Cur, demonstrando uma captação eficaz.

Nos estudos in vivo, o acúmulo de IR-780 ocorrido nas células tumorais foi maior nos grupos associados ao nanocarreador quando comparado aos grupos tratados com IR-780 livre, fator ligado ao maior tempo de circulação da droga associada ao nanocarreador e ao efeito de permeabilidade e retenção aumentada. Os estudos in vivo mostraram ainda um efeito anticâncer maior nos grupos tratados com NP/IR+Cur associado à radioterapia e terapia fototérmica, em comparação às mesmas terapias isoladamente (Zhang, 2019).

A distribuição dos estudos elegíveis conforme a organização da análise crítica das informações, podem ser visualizados no Quadro 1.

Quadro 1. Características incluídas no estudo.

\begin{tabular}{|c|c|c|c|}
\hline Autor & Estudo & Descrição do Nanocarreador / Droga & Complicações associadas \\
\hline Panda, 2020 & In vitro & $\begin{array}{l}\text { Nanocarreador de ferrita de cobalto } \\
\text { carregado com docetaxel (DTX) (CF-CP) }\end{array}$ & $\begin{array}{l}\text { Não foram reportadas complicações significativas associadas à } \\
\text { linhagem de células MCF-7 (câncer de mama). }\end{array}$ \\
\hline Kumar, 2018 & In vitro & $\begin{array}{l}\text { Doxorrubicina (DOX) carregada por } \\
\text { Triptofano-(N-acetilglucosamina) }[\mathrm{PTN}]\end{array}$ & $\begin{array}{l}\text { A liberação prolongada do fármaco observada pode ser atribuída à } \\
\text { presença de triptofano e NAG que causam maior impedimento } \\
\text { estérico. }\end{array}$ \\
\hline $\begin{array}{l}\text { Azandaryan, } \\
2017\end{array}$ & In vitro & $\begin{array}{l}\text { Letrozol (LTZ) carregado por FA- } \\
\text { PLN(polímero acoplado a FA } \\
\text { nanocarreador híbrido de lipídio). }\end{array}$ & $\begin{array}{l}\text { A ausência de um grupo funcional em sua cadeia o torna menos eficaz } \\
\text { em aplicações específicas. NPs Poliméricas }\end{array}$ \\
\hline Abarazi, 2018 & In vitro & $\begin{array}{l}\mathrm{CS}(\text { quitosana })+\text { Bio-MOF(estrutura } \\
\begin{array}{l}\text { biológica metálica-orgânica) / DOX } \\
\text { (doxorubicina) }\end{array}\end{array}$ & $\begin{array}{l}\text { Em alguns casos foram encontradas interações exageradamente fortes } \\
\text { entre as moléculas nanocerregadas e as células resultando em } \\
\text { variações estruturais e quimisorção. }\end{array}$ \\
\hline Kumari, 2017 & $\begin{array}{l}\text { In vivo/ } \\
\text { In vitro }\end{array}$ & $\begin{array}{l}\text { mPEG-PLA-Ch (Colesterol ácido } \\
\text { conjugado metóxi polietileno glicol- } \\
\text { polilactico) / CUR (Curcumina) }\end{array}$ & $\begin{array}{l}\text { O tratamento CUR-mPEG-PLA-Ch foi mais eficaz em matar células } \\
\text { cancerosas, exceto no ponto de tempo de } 24 \mathrm{~h} \text { na linha celular B16F10. } \\
\text { Isto pode ser devido à liberação incompleta do fármaco carregado da } \\
\text { formulação, resultando em uma menor eficácia no tratamento. Além } \\
\text { disso, estudos anteriores por outros grupos indicaram que o valor de } \\
\text { IC50 variou em diferentes linhas de células com o mesmo tratamento. }\end{array}$ \\
\hline
\end{tabular}




\begin{tabular}{|c|c|c|c|}
\hline $\mathrm{Li}, 2019$ & $\begin{array}{l}\text { In vivo/ } \\
\text { In vitro }\end{array}$ & $\begin{array}{l}\text { PTL (Ácido dehidrotumulósico liposomal) / } \\
\text { DOX (doxorubicina) + Extrato de Poria } \\
\text { cocos }\end{array}$ & $\begin{array}{l}\text { A proporção de PT para DOX em lipossomas pode afetar a eficácia } \\
\text { dos lipossomas carregados. In vitro os resultados de citotoxicidade } \\
\text { demonstraram que, ao otimizar as proporções de drogas, a co-entrega } \\
\text { de lipossomas pode resultar em melhor eficácia antitumoral. }\end{array}$ \\
\hline $\begin{array}{l}\text { Kobylinska, } \\
2018\end{array}$ & $\begin{array}{l}\text { In vivol } \\
\text { in vitro }\end{array}$ & $\begin{array}{l}\text { Poli(VEP-co-GMA)-graft-mPEG } \\
\text { consistindo de um esqueleto de copolímero } \\
\text { 5-(tert-butilperoxi)-5-metilex-1-en-3-ino } \\
\text { (VEP, indicado por "l") e glicidil metacilato } \\
\text { (GMA, indicado por "m"), e cadeias laterais } \\
\text { de PEG enxertadas (PEG indicada por " } n \text { ") }\end{array}$ & $\begin{array}{l}\text { Os ratos recebendo o PNC demonstraram uma elevação significante } \\
\text { de } 55,4 \% \text { de atividade da ALP no } 20^{\circ} \text { dia, quando comparados com os } \\
\text { ratos do grupo controle. A atividade de outras enzimas foi próxima dos } \\
\text { valores normais, com pequenos desvios quando comparado ao grupo } \\
\text { controle: atividade da GGT foi diminuída em } 10 \% \text {, ALT em } 18 \% \text {, } \\
\text { LDH em } 33 \% \text { e a CK em } 16 \% \text {, enquanto a atividade da } \alpha \text {-amilase foi } \\
\text { aumentada em } 17 \% \text {. }\end{array}$ \\
\hline Yu, 2020 & $\begin{array}{l}\text { In vivol } \\
\text { in vitro }\end{array}$ & $\begin{array}{l}\text { HA (ácido hialurônico)/ DOX } \\
\text { (doxorrubicina) }+ \text { CDDP (cisplatina) }\end{array}$ & $\begin{array}{l}\text { Não foram encontradas complicações relacionadas ao uso do } \\
\text { HA/DOX+CDDP no tratamento das linhagens de células neoplásicas } \\
\text { utilizadas no estudo. }\end{array}$ \\
\hline Tingting, 2018 & $\begin{array}{l}\text { In vivol } \\
\text { in vitro }\end{array}$ & $\begin{array}{l}\text { (CSOSA) Quitosana ácido-exertado } \\
\text { esteárico oligosacarídeo / DOX } \\
\text { (doxorubicina) }\end{array}$ & $\begin{array}{l}\text { O tratamento prolongado e contínuo de CSOSA / DOX pode } \\
\text { apresentar a possibilidade de tornar as células resistentes aos } \\
\text { medicamentos. }\end{array}$ \\
\hline $\begin{array}{l}\text { Vinothini, } \\
2019\end{array}$ & $\begin{array}{l}\text { In vitrol } \\
\text { in vivo }\end{array}$ & $\begin{array}{l}\text { Nanocarreador de óxido de grafeno-acrilato } \\
\text { de metila (GO-g-MA) carregado de } \\
\text { paclitaxel (PTX) }\end{array}$ & $\begin{array}{l}\text { Não foram feitos ensaios de citotoxicidade em células saudáveis de } \\
\text { PBMC para melhor avaliação. }\end{array}$ \\
\hline Rahimi, 2019 & In vitro & $\begin{array}{l}\text { Superfície de nanopartículas magnéticas de } \\
\text { Fe3O4 cobertas por Macrociclo de } \\
\text { calix[4]areno anfotérico (calix) }\end{array}$ & $\begin{array}{l}\text { Ao aumentar a concentração do nanocarreador, a viabilidade celular } \\
\text { foi diminuída, o que atribuiu à sedimentação do nanocarreador em } \\
\text { anexo causando hipóxia (deficiência na quantidade de oxigênio que } \\
\text { chega aos tecidos), }\end{array}$ \\
\hline Pei, 2020 & $\begin{array}{l}\text { In vitro } \\
\text { in vivo }\end{array}$ & $\begin{array}{l}\text { Nanocompósito de óxido de grafeno } \\
\text { PEGuilado (pGO) duplamente carregado } \\
\text { com cisplatina (Pt) e doxorrubicina (DOX) }\end{array}$ & $\begin{array}{l}\text { Uma seção cardíaca de amostra tratada com Pt / DOX mostrou } \\
\text { alterações marcantes de danos, incluindo congestão miocárdica e } \\
\text { hipertrofia de cardiomiócitos, indicando uma quantidade considerável } \\
\text { de DOX acumulada no tecido cardíaco, causando toxicidade cardíaca } \\
\text { evidente. Além disso, as alterações histopatológicas óbvias do baço } \\
\text { foram encontradas em camundongos tratados com Pt / DOX, } \\
\text { sugerindo o acúmulo de Pt. }\end{array}$ \\
\hline Zhang, 2019 & $\begin{array}{l}\text { In vitro } \\
\text { In vivo }\end{array}$ & 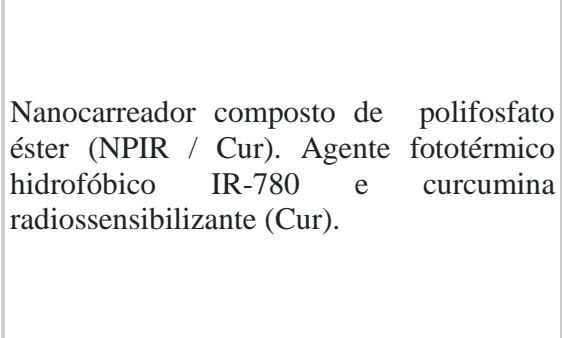 & $\begin{array}{l}\text { Nenhuma citotoxicidade perceptível das formulações de } \\
\text { nanopartículas foi observada em qualquer uma das linhas de células, } \\
\text { mesmo em uma concentração de até } 800 \mu \mathrm{g} / \mathrm{mL} \text {. Um fenômeno de } \\
\text { enriquecimento do fígado e baço foi observado nos grupos NPIR / Cur } \\
\text { e NPIR 24 horas após a injeção, o qual foi relacionado à depuração do } \\
\text { sangue pelo sistema reticuloendotelial. O tratamento com NPIR / Cur } \\
\text { ou NPIR de tamanho nanométrico aumentou notavelmente a } \\
\text { deposição de IR-780 no tecido tumoral em comparação com IR-780 } \\
\text { livre. }\end{array}$ \\
\hline $\begin{array}{l}\text { Akbarzadeh, } \\
2021\end{array}$ & In vitro & $\begin{array}{l}\text { Niossoma-alginato de cálcio como um nano } \\
\text { transportador que continha curcumina }\end{array}$ & $\begin{array}{l}\text { O aumento da concentração das amostras mencionadas levou a uma } \\
\text { diminuição da viabilidade celular, indicando diferenças significativas } \\
\text { na toxicidade em todas as amostras. As amostras armazenadas a } 4 \pm 2 \\
\text { 'C têm liberações mais baixas e mais lentas devido ao declínio da } \\
\text { mobilidade da membrana nesta temperatura. }\end{array}$ \\
\hline $\mathrm{Hu}, 2016$ & In vitro & $\begin{array}{l}\text { Nanocarreador à base de quitosana de } \\
\text { dissulfeto-ligado (CS-ss-SA) para a } \\
\text { coadministração de paclitaxel (PTX) e } \\
\text { siRNA específico de Bcl-2 (siBcl-2) }\end{array}$ & $\begin{array}{l}\text { O tempo / sequência otimizado de liberação dos agentes citotóxicos e } \\
\text { siRNA do sistema, que é um fator chave para alcançar o melhor } \\
\text { resultado sinérgico, não é bem compreendido. }\end{array}$ \\
\hline Meng, 2017 & $\begin{array}{l}\text { In vitro/ } \\
\text { in vivo }\end{array}$ & $\begin{array}{l}\text { Conjugado de glicolipídio redox- } \\
\text { responsivo e siRNA (CSO-ss-SA/siRNA) } \\
\text { encapsulando doxorrubicina (DOX) }\end{array}$ & $\begin{array}{l}\text { Degradação da ribonuclease (RNase), eliminação, pior permeabilidade } \\
\text { e aprisionamento do endossomo. }\end{array}$ \\
\hline
\end{tabular}




\begin{tabular}{|c|c|c|c|}
\hline Karim, 2019 & $\begin{array}{l}\text { In vivol } \\
\text { In vitro }\end{array}$ & Sulfeto de Estrôncio & $\begin{array}{l}\text { siRNA não modificado é propenso à degradação de nuclease, } \\
\text { depuração fagocítica, interações com proteínas séricas e depuração } \\
\text { renal. Além disso, o siRNA também lida com o microambiente } \\
\text { anormal tumoral (TME), incluindo a matriz extracelular densa (ECM), } \\
\text { e vazamentos e vasos heterogêneos que contribuem para o aumento da } \\
\text { pressão do fluido intersticial (IFP). }\end{array}$ \\
\hline $\begin{array}{l}\text { Abayaweera, } \\
2017\end{array}$ & In vitro & $\begin{array}{l}\text { Ferro e enxofre dos nanocarreadores de } \mathrm{Fe} / \\
\mathrm{Fe}_{3} \mathrm{O}_{4}\end{array}$ & $\begin{array}{l}\text { Um fator de } 2,2 \text { entre as viabilidades celulares determinadas para } \\
\text { células cancerígenas } 4 \mathrm{~T} 1 \text { e células de fibroblastos murinos (controle) } \\
\text { não é suficiente para sugerir o início de estudos clínicos. }\end{array}$ \\
\hline $\begin{array}{l}\text { Shaarani, } \\
2017\end{array}$ & In vitro & Pluronic F68 e F127/ Timoquinona (TQ) & $\begin{array}{l}\text { A porcentagem máxima de TQ liberada do sistema de controle foi de } \\
51 \% \text {, enquanto a liberação de micelas encapsuladas de PF127 foi de } \\
62 \% \text { em } 48 \text { h. Relatórios adicionais são necessários para explorar as } \\
\text { possíveis vias de degradação e os produtos resultantes para ter um } \\
\text { estudo mais abrangente sobre as flutuações observadas no perfil de } \\
\text { solubilidade. }\end{array}$ \\
\hline
\end{tabular}

Fonte: Autores (2021).

\section{Conclusão}

A correlação entre nanocarreadores e o tratamento do câncer de mama é bem esclarecida nos estudos. Como limitações, foi encontrado dificuldades em encontrar estudos nas bases de dados que avaliassem esse tratamento em pacientes diagnosticados com CM, pois os estudos in vitro e com animais ainda são predominantes para garantir o sucesso na prática clínica. No entanto, foi possível observar que existe eficácia das drogas utilizadas nos diferentes tipos de nanocarreadores para a redução das células tumorais com minimização da toxicidade e liberação do medicamento na região específica do tumor, implicando que esse tratamento tem grande utilidade nas práticas clínicas.

Essa eficácia deve ser melhor estudada para esclarecer as vias farmacológicas e fisiopatológicas desse tratamento que podem estar relacionadas aos tipos de células tumorais do CM, possibilitando mais estudos que aprimorem esses tratamentos para pacientes diagnosticados com câncer de mama. Além disso, salienta-se a necessidade de outros estudos envolvendo diversos tipos de células tumorais, avaliando sua aplicabilidade, sobretudo em pacientes com quadro de metástase avançada.

\section{Agradecimentos}

O presente trabalho foi realizado com o apoio da disciplina de metodologia científica da Universidade Católica de Pernambuco.

\section{Referências}

Abazari, R., Mahjoub, A. R., Ataei, F., Morsali, A., Carpenter-Warren, C. L., Mehdizadeh, K., \& Slawin, A. M. (2018). Chitosan immobilization on bio-MOF nanostructures: a biocompatible pH-responsive nanocarrier for doxorubicin release on MCF-7 cell lines of human breast cancer. Inorg Chem, 57(21), 1336413379.

Akbarzadeh, I., Shayan, M., Bourbour, M., Moghtaderi, M., Noorbazargan, H., Eshrati Yeganeh, F., \& Tahriri, M. (2021). Preparation, Optimization and InVitro Evaluation of Curcumin-Loaded Niosome@ calcium alginate nanocarrier as a new approach for breast cancer treatment. Biology, 10(3), 173-200.

Azandaryani, A. H., Kashanian, S., \& Derakhshandeh, K. (2017). Folate conjugated hybrid nanocarrier for targeted letrozole delivery in breast cancer treatment. Pharm Res, 34(12), 2798-2808.

Bahrami, B., Hojjat-Farsangi, M., Mohammadi, H., Anvari, E., Ghalamfarsa, G., Yousefi, M., \& Jadidi-Niaragh, F. (2017). Nanoparticles and targeted drug delivery in cancer therapy. Immunol Lett, 190, 64-83.

Draz, M. S., Fang, B. A., Zhang, P., Hu, Z., Gu, S., Weng, K. C., \& Chen, F. F. (2014). Nanoparticle-mediated systemic delivery of siRNA for treatment of cancers and viral infections. Theranostics, 4(9), 872-892.

Estrela, C. (2018). Metodologia científica: ciência, ensino, pesquisa. Artes Médicas. 
INSTITUTO NACIONAL DO CÂNCER. Câncer de mama, 2021. Disponível em: https://www.inca.gov.br/tipos-de-cancer/cancer-de-mama. Acesso em: 29 abr. 2021

Jones, S. E. (2008). Metastatic breast cancer: the treatment challenge. Clin. Breast cancer, 8(3), 224-233.

Kobylinska, L., Patereha, I., Finiuk, N., Mitina, N., Riabtseva, A., Kotsyumbas, I., \& Vari, S. G. (2018). Comb-like PEG-containing polymeric composition as low toxic drug nanocarrier. Cancer nanotechnol, 9(1), 1-13.

Kumar, P., Paknikar, K. M., \& Gajbhiye, V. (2018). A robust pH-sensitive unimolecular dendritic nanocarrier that enables targeted anti-cancer drug delivery via GLUT transporters. Colloid Surface B, 171, 437-444.

Kumari, P., Muddineti, O. S., Rompicharla, S. V. K., Ghanta, P., BBN, A. K., Ghosh, B., \& Biswas, S. (2017). Cholesterol-conjugated poly (D, L-lactide)-based micelles as a nanocarrier system for effective delivery of curcumin in cancer therapy. Drug Deliv, 24(1), 209-223.

Lamb, R., Bonuccelli, G., Ozsvári, B., Peiris-Pagès, M., Fiorillo, M., Smith, D. L., Bevilacqua G., Mazzanti, C. M., McDonnel, L. A., Naccarato, A. G., Chiu, M., Wynne, L., Martinez-Outschoorn, U. E., Sotgia, F., \& Lisanti, M. P. (2015). Mitochondrial mass, a new metabolic biomarker for stem-like cancer cells: Understanding WNT/FGF-driven anabolic signaling. Oncotarget, 6(31), 30453-71.

Li, Y., Li, X., Lu, Y., Chaurasiya, B., Mi, G., Shi, D., Chen, D., Webster, T. J., Tu, J., \& Shen, Y. (2020). Co-delivery of Poria cocos extract and doxorubicin as an 'all-in-one'nanocarrier to combat breast cancer multidrug resistance during chemotherapy. $N B M, 23,938-941$.

Lu, J., Steeg, P. S., Price, J. E., Krishnamurthy, S., Mani, S. A., Reuben, J., Cristofanilli, M., Dontu, G., Bidaut, L., Valero, V., N. Hortobagyi, G., \& Yu, D. (2009). Breast cancer metastasis: challenges and opportunities. Cancer Res, 69(12), 4951-4953

Ma, H., Yang, X., Ke, J., Wang, C., Peng, L., Hu, F., \& Yuan, H. (2020). Smart assembled human serum albumin nanocarrier enhanced breast cancer treatment and antitumor immunity by chemo-photothermal therapy. ACS Biomater-Sci Eng, 6(5), 3217-3229.

Maji, R., Dey, N. S., Satapathy, B. S., Mukherjee, B., \& Mondal, S. (2014). Preparation and characterization of Tamoxifen citrate loaded nanoparticles for breast cancer therapy. Int J Nanomedicine, 9, 3107-3118.

Meng, T., Lu, B., Shao, S., Yuan, M., Liu, X., Yuan, H., Huang, X., \& Hu, F. (2017). Sequential therapy with redox-responsive glucolipid nanocarrier separately delivering siRNA and doxorubicin to overcome multidrug resistance. Int J Pharm, 534(1-2), 368-377.

Meng, T., Qiu, G., Hong, Y., Yuan, M., Lu, B., Wu, J., Yuan, H., \& Hu, F. (2019). Effect of chitosan based glycolipid-like nanocarrier in prevention of developing acquired drug resistance in tri-cycle treatment of breast cancer. Int J Pharm, 555, 303-313.

Ouzzani, M., Hammady, H., Fedorowicz, Z., \& Elmagarmid, A. (2016). Rayyan—a web and mobile app for systematic reviews. Syst Rev, 5(1), 1-10.

Padayachee, J., Daniels, A., Balgobind, A., Ariatti, M., \& Singh, M. (2020). HER-2/neu and MYC gene silencing in breast cancer: therapeutic potential and advancement in nonviral nanocarrier systems. Nanomedicine, 15(14), 1437-1452.

Panda, J., Satapathy, B. S., Mandal, B., Sen, R., Mukherjee, B., Sarkar, R., \& Tudu, B. (2021). Anticancer potential of docetaxel-loaded cobalt ferrite nanocarrier: an in vitro study on MCF-7 and MDA-MB-231 cell lines. J Microencapsul, 38(1), 36-46.

Pei, X., Zhu, Z., Gan, Z., Chen, J., Zhang, X., Cheng, X., Wan, Q., \& Wang, J. (2020). PEGylated nano-graphene oxide as a nanocarrier for delivering mixed anticancer drugs to improve anticancer activity. Sci Rep, 10(1), 1-15.

Pourjavadi, A., Amin, S. S., \& Hosseini, S. H. (2018). Delivery of hydrophobic anticancer drugs by hydrophobically modified alginate based magnetic nanocarrier. Ind Eng Chem Res, 57(3), 822-832.

Rahimi, M., Karimian, R., Noruzi, E. B., Ganbarov, K., Zarei, M., Kamounah, F. S., Yousefi, B., Bastami, M., Yousefi M., \& Kafil, H. S. (2019). Needle-shaped amphoteric calix [4] arene as a magnetic nanocarrier for simultaneous delivery of anticancer drugs to the breast cancer cells. Int J Nanomedicine, 14, 26192636.

Shaarani, S., Hamid, S. S., \& Kaus, N. H. M. (2017). The Influence of pluronic F68 and F127 nanocarrier on physicochemical properties, in vitro release, and antiproliferative activity of thymoquinone drug. Pharmacogn Res, 9(1), 12-20.

Siegel, R., DeSantis, C., Virgo, K., Stein, K., Mariotto, A., Smith, T., Fedewa, S., Lin, C., Leach C., Cannady, R., Cho, H., Scoppa, S., Hachey, M., Kirch, R., Jemal, A., \& Ward, E. (2012). Cancer treatment and survivorship statistics, 2012. CA: Cancer J Clin, 62(4), 220-241.

Vinothini, K., Rajendran, N. K., Ramu, A., Elumalai, N., \& Rajan, M. Internat. J. Pharm. (2019). Folate receptor targeted delivery of paclitaxel to breast cancer cells via folic acid conjugated graphene oxide grafted methyl acrylate nanocarrier. Biomed Pharmacother, 110, 906-917.

Wolrd Health Organization (2021). Breast cancer 2020. https://www.who.int/news-room/fact-sheets/detail/breast-cancer\#: :text=The\%20objective \%20of\%20the\%20W HO,globally\%20between\%202020\%20and\%202040.

Yu, T., Li, Y., Gu, X., \& Li, Q. (2020). Development of a Hyaluronic Acid-Based Nanocarrier Incorporating Doxorubicin and Cisplatin as a PH-Sensitive and CD44-Targeted Anti-Breast Cancer Drug Delivery System. Front Pharmacol, 11, 1370. 